\title{
Políticas públicas para redução de assimetrias e a pós-graduação na Região da
}

\section{Amazônia Legal/Brasil}

\author{
Public policies to asymmetries and postgraduate studies in the Legal Amazon Region/Brazil \\ Políticas públicas para reducir assimetrias y estúdios de postgrado em lá Región Legal
}

Amazónia/Brasil

Recebido: 09/10/2021 | Revisado: 19/10/2021 | Aceito: 22/10/2021 | Publicado: 24/10/2021

Daniela Côco

ORCID: https://orcid.org/0000-0003-4340-0242 Universidade Federal do Rio Grande do Sul, Brasil E-mail: danielacocobqi@gmail.com

Livio Amaral

ORCID: https://orcid.org/0000-0002-4732-5502 Universidade Federal do Rio Grande do Sul, Brasil E-mail: amaral@if.ufrgs.br

\begin{abstract}
Resumo
Circunscrevendo o campo educacional, este artigo atualiza, até o ano de 2020, as informações sobre os principais programas da Coordenação de Aperfeiçoamento de Pessoal de Nível Superior - CAPES voltados à redução de assimetrias no Sistema Nacional de Pós-Graduação - SNPG brasileiro, e apresenta um panorama da pós-graduação na Amazônia Legal - Brasil, visando contribuir com as reflexões sobre as assimetrias no SNPG e as abordagens das estratégias de indução. Nesse intuito, foram exploradas informações disponibilizadas no sítio institucional da CAPES e na Plataforma Sucupira. Como resultado, verificou-se a evolução da pós-graduação na Amazônia Legal, que concentrava, no ano de 2020, 421 programas; a maior parte deles recentes, emergentes e em consolidação, o que evidencia também a importância de políticas de consolidação. Observou-se que apesar das diversas políticas públicas voltadas à consolidação da pós-graduação e redução de assimetrias, ao longo do tempo, há ainda diferenças acentuadas entre as regiões do Brasil, especialmente, na área compreendida pela Amazônia Legal.
\end{abstract}

Palavras-chave: Pós-graduação; Amazônia Legal; Assimetrias; Políticas públicas.

\begin{abstract}
Circumscribing the educational field, this article updates, until 2020, information on the main CAPES programs aimed at reducing asymmetries in the Brazilian National Graduate System (SNPG) and the panorama of postgraduate studies in the Legal Amazon - Brazil, aiming at contribute to the reflections on the asymmetries in the SNPG and the approaches of induction strategies. For this purpose, information available on the CAPES institutional website and on the Sucupira Platform were explored. As a result, there was an evolution of postgraduate studies in the Legal Amazon, which in 2020 concentrated 421 programs; most recent, emerging and in consolidation, highlighting the importance of consolidation policies. It was observed that despite the various public policies aimed at consolidating postgraduate studies and reducing asymmetries, over time, there are still marked differences between the regions of the country, especially in the area comprised by the Legal Amazon.
\end{abstract}

Keywords: Post-graduation; Legal Amazon; Asymmetries; Public policies.

\section{Resumen}

Circunscribiendo el campo educativo, este artículo actualiza, hasta 2020, la información sobre los principales programas de la CAPES orientados a reducir las asimetrías en el Sistema Nacional de Posgrado - SNPG de Brasil y presenta un panorama de estudios de posgrado en la Amazonía Legal - Brasil, con el objetivo de contribuir a las reflexiones sobre asimetrías en el SNPG y enfoques de estrategias de inducción. Para ello, se exploró la información disponible en el sitio web institucional de CAPES y en la Plataforma Sucupira. Como resultado, hubo una evolución de los estudios de posgrado en la Amazonia Legal, que en 2020 concentró 421 programas; la mayoría de ellos recientes, emergentes y en consolidación, lo que también destaca la importancia de las políticas de consolidación. Se observó que a pesar de las diversas políticas públicas orientadas a consolidar los estudios de posgrado y reducir las asimetrías, a lo largo del tiempo, aún existen marcadas diferencias entre las regiones del país, especialmente en el área que comprende la Amazonía Legal.

Palabras clave: Posgrado; Amazonía Legal; Assimetrias; Políticas públicas. 


\section{Introdução}

A educação é o mais potente e impulsor mecanismo de desenvolvimento e transformação social, ganhando especificidades conforme os níveis, etapas e modalidades, sobretudo no que se refere às políticas de investimentos, de acompanhamento e avaliação. Nesse contexto, focalizamos a pós-graduação, no quadro de reconhecimento da importância do seu papel na formação de recursos humanos e disseminação do conhecimento e de atenção ao seu desenvolvimento na realidade brasileira, marcado pela necessidade de superar a concentração nas grandes cidades e nas regiões sul e sudeste (CAPES, 2010).

Com isso em vistas, a Coordenação de Aperfeiçoamento de Pessoal de Nível Superior - CAPES), Fundação vinculada ao Ministério da Educação - MEC do Brasil, atua na expansão e consolidação da pós-graduação stricto sensu (mestrado e doutorado) em todos os estados do país, por meio de políticas públicas de indução e fomento. Além de ser responsável por avaliações regulares e periódicas da pós-graduação nacional, com foco em manter o padrão de qualidade dos cursos ofertados no âmbito do Sistema Nacional de Pós-Graduação - SNPG brasileiro.

Quando se faz um recorte da pós-graduação, é possível constatar assimetrias na distribuição dos programas de pósgraduação pelo Brasil, sendo a região da Amazônia Legal (estados do Acre, Amapá, Amazonas, Mato Grosso, Pará, Rondônia, Roraima, Tocantins e parte do estado do Maranhão) portadora do menor número de programas em comparação com as demais regiões (Rodrigues, 2014; Plataforma Sucupira, 2020). A Amazônia Legal por ser uma região estratégica, onde há grandes riquezas, é foco de várias políticas governamentais, dentre elas políticas públicas de indução para a criação de novos programas de pós-graduação e para a consolidação dos já existentes na região, com o intuito de estimular as pesquisas e consequente o desenvolvimento local.

Nesse sentido, com o propósito de estudar o cenário contemporâneo da pós-graduação na Amazônia Legal, este artigo analisa e debate as principais políticas públicas pertinentes à região, valendo-se de dados quantitativos, informações e tendo como pano de fundo os Planos Nacionais de Pós-Graduação - PNPG e a atuação da CAPES até o ano de 2020.

\section{Metodologia}

Para avançar na descrição das políticas públicas educacionais, conduzidas pela CAPES, para redução de assimetrias no SNPG e na análise do panorama da pós-graduação na região da Amazônia Legal, adotamos para a apreensão dos dados a técnica de análise documental. Essa técnica é frequentemente empregada na verificação e acesso a fontes de informação, possibilitando a geração de novos conhecimentos e distintas formas de interpretar os fenômenos (Sá-Silva, Almeida \& Guidani, 2009).

A pesquisa considerou conjuntamente elementos provenientes dos métodos qualitativo e quantitativo. Para captação das informações relacionadas as políticas públicas voltadas a redução de assimetrias utilizamos os dados disponibilizados no site institucional da CAPES e nos Relatórios de Gestão da CAPES, 2008, 2009, 2011, 2015 e 2016. Para a descrição do panorama da pós-graduação na Amazônia Legal, considerando o ano de 2020, servimos dos dados constantes na Plataforma Sucupira (https://sucupira.capes.gov.br/sucupira/) e no Sistema de Informações Georreferenciadas - GEOCAPES (https://geocapes.capes.gov.br/geocapes/), bases de acesso público gerenciadas pela CAPES.

\section{Planos Nacionais de Pós-Graduação}

Em uma dimensão histórica, cabe registrar que a pós-graduação no Brasil foi formalizada em 1965, com o Parecer $\mathrm{n}^{\circ}$ 977/65 do Conselho Federal de Educação - CFE, pelo qual foi regulamentada seus níveis (de mestrado e doutorado) e sua função (Patrus et al., 2018). Para o seu desenvolvimento e sua ampliação, foram aplicadas as diretrizes e orientações previstas nos Planos 
Nacionais de Pós-Graduação, documentos de caráter norteador das políticas públicas de indução e fomento à pós-graduação no Brasil.

Em 1974 o Decreto n 73.411 instituiu o Conselho Nacional de Pós-Graduação, no então Ministério da Educação e Cultura. Dos trabalhos desse Conselho derivou o I Plano Nacional de Pós-Graduação (I PNPG - 1975/1979), com as diretrizes de institucionalizar o sistema, elevar os padrões existentes e planejar sua expansão (França, 2012; Carvalho \& Silva, 2017).

O II Plano Nacional de Pós-Graduação de 1982/1985, foi proposto em um contexto de recessão econômica. Aparentemente, mais simples que o I PNPG - 1975/1979, aquele focou seus objetivos na busca da qualidade, indicando o aperfeiçoamento dos sistemas de avaliação da pós-graduação e a revisão dos mecanismos que determinavam o apoio e o financiamento às instituições (França, 2012; Carvalho \& Silva, 2017).

O III Plano Nacional de Pós-Graduação vigorou de 1986/1989, focalizando o diagnóstico de que o País não possuía na época um quantitativo de cientistas suficiente para atingir, rapidamente, a máxima capacitação científica e tecnológica. Diante disso, o plano tinha como objetivos o aperfeiçoamento dos cursos de pós-graduação, o estabelecimento da pesquisa nas universidades e a inserção da pós-graduação no sistema de Ciência e Tecnologia, juntamente com o setor produtivo (França, 2012 \& Carvalho, Silva, 2017).

Nos anos de 1990 não houve a formalização de um IV Plano Nacional de Pós-Graduação, devido a diversas questões como restrição orçamentária e falta de articulação entre as agências de fomento existentes. Entretanto, importantes ações foram implantadas nesse período como a expansão do sistema, a diversificação do modelo de pós-graduação, alterações no processo de avaliação e inserção internacional da pós-graduação (França, 2012; Carvalho \& Silva, 2017).

Um novo plano foi oficializado nos anos 2000, com vigência de cinco anos: o Plano Nacional de Pós-Graduação de 2005/2010. O objetivo desse plano era o crescimento equânime do SNPG, por intermédio das seguintes diretrizes: estabilidade e indução, estratégias para melhoria do sistema, financiamento e sustentabilidade, novos modelos, políticas de cooperação internacional e de formação de recursos humanos no exterior e, avaliação e qualidade (França, 2012; Carvalho \& Silva, 2017).

O último plano em vigor, o Plano Nacional de Pós-Graduação de 2011/2020, apresenta como alicerce as linhas: expansão do Sistema Nacional de Pós-Graduação, criação de uma agenda nacional de pesquisa, aperfeiçoamento da avaliação, a multi/interdisciplinaridade e o apoio a outros níveis de ensino (Kato \& Ferreira, 2016).

A análise do conjunto dos planos permite compor um panorama da pós-graduação, considerando as mudanças contextuais e históricas e os desafios que foram enfrentados até o presente, tanto no conjunto da realidade nacional, quanto nas particularidades dos contextos regionais. A existência de assimetrias no sistema de pós-graduação brasileiro é mencionada em diversos documentos, chegando a ganhar um tópico próprio no Plano 2011/2020. Nesse Plano, a abordagem das assimetrias contempla as diferenças entre estados, regiões, mesorregiões e áreas de conhecimento.

\section{A CAPES}

A CAPES foi criada em 11 de julho de 1951, por meio do Decreto nº 29.741 da Presidência da República, no âmbito do Ministério da Educação e Saúde Pública. Inicialmente, com o nome de Campanha Nacional de Aperfeiçoamento de Pessoal de Nível Superior, objetivava a formação de pessoal especializado para atender às necessidades de instituições públicas e privadas. É importante registrar que essa era uma época de intensa industrialização no Brasil, em que se evidenciava a necessidade de formação de especialistas nas mais diversas áreas (Martins et al., 2012).

A missão da CAPES destaca sua contribuição na expansão e consolidação da pós-graduação stricto sensu (mestrado e doutorado) em todos os estados brasileiros (BRASIL, 2008). Sua história se identifica e se integra ao próprio crescimento da pós-graduação, uma vez que é agente na formulação e aplicação dos Planos Nacionais de Pós-Graduação. Dentre suas linhas de 
atuação destacamos a avaliação, o acesso à produção científica e o fomento e estabelecimento de políticas públicas para a pósgraduação stricto sensu.

Desde 1976, a CAPES realiza, por meio de sua Diretoria de Avaliação - DAV, a avaliação do sistema brasileiro de pósgraduação, baseada na premissa de ampla participação da comunidade acadêmico-científica, por meio da colaboração de consultores ad hoc, membros da academia, em diferentes etapas. A avaliação é essencial para assegurar e manter a qualidade dos cursos de mestrado e doutorado no Brasil, certificando a qualidade da pós-graduação brasileira e sendo referência para as políticas públicas de investimentos, notadamente na forma da concessão de bolsa e de fomento à pesquisa.

A primeira certificação da qualidade da pós-graduação brasileira consiste na Avaliação das Propostas de Cursos Novos - APCN. As instituições de ensino superior que desejam a implantação de um novo curso de pós-graduação, submetem propostas ao processo de APCN. Após análises técnicas e administrativas o novo curso recebe o status de "aprovado" ou "não aprovado" (CAPES, 2021).

Os cursos de mestrado e doutorado em andamento, passam pela avaliação periódica de permanência, de 4 em 4 anos (quadrienal), com o objetivo de garantir a melhoria contínua da qualidade. Na avaliação de permanência, os programas de pósgraduação fornecem informações relacionadas ao desempenho do programa e estas são avaliadas por comissões formadas por profissionais de reconhecida competência técnica e científica, que analisam a evolução dos programas. Ao final de todo o processo de avaliação, que envolve diversas etapas, os programas recebem notas que variam de 1 a 7 . As notas 6 e 7 são destinadas, exclusivamente, aos PPGs com oferta de doutorado e correspondem a programas com alto padrão internacional de desempenho, considerados referências para as áreas; a nota 5 corresponde à nota máxima admitida para PPGs que ofertam apenas o nível de mestrado e a nota 3 corresponde ao padrão mínimo de qualidade estabelecido para a pós-graduação stricto sensu. Programas com notas muito baixas são desativados. Enfatiza-se que a avaliação tem centralidade para as ações de fomento da CAPES, subsidiando normativas relativas ao financiamento dos diversos programas de fomento da instituição, com repasses atrelados à nota atribuída aos programas de pós-graduação. (CAPES, 2021).

$\mathrm{Na}$ atuação para o acesso à produção científica, a CAPES é detentora do Portal de Periódicos, uma das políticas públicas do governo brasileiro para acesso à informação científica e tecnológica, de maior importância e abrangência. Criado, em 11 de novembro de 2000, o Portal de Periódicos tornou-se uma biblioteca virtual que reúne e disponibiliza às instituições de ensino e pesquisa no país o melhor da produção científica nacional e internacional, possuindo atualmente um acervo de mais de 45 mil títulos com texto completo (Miranda et al., 2015).

O Portal de Periódicos da CAPES foi desenvolvido devido as dificuldades no acesso das bibliotecas brasileiras à informação científica internacional, uma vez que seus acervos, à época, eram mantidos por meio da compra de periódicos impressos para cada uma das universidades do sistema superior de ensino federal. Com o surgimento dos periódicos eletrônicos e a instituição do Portal, os pesquisadores brasileiros vinculados às instituições beneficiárias passaram a acessar simultaneamente o mesmo artigo em qualquer região do País, sem restrições geográficas e de fuso horário (Miranda et al., 2015).

Assim, o Portal foi redutor das desigualdades regionais relacionadas ao acesso à Informação em Ciência e Tecnologia, por promover o acesso à informação em tempo real, disponibilizando aos pesquisadores acesso à produção científica nacional e internacional atualizada, propiciando avanços na pós-graduação brasileira. Destaca-se que o Portal foi um dos únicos modelos de consórcio de bibliotecas no mundo inteiramente financiado pelo governo. (Miranda et al., 2015).

\section{Programas para Redução de Assimetrias Regionais}

No primeiro ano do último PNPG (2011/2020), importantes iniciativas voltadas ao desenvolvimento regional já estavam em execução. Analisando os programas ativos no ano de 2011 e os posteriormente criados até o ano de 2020, foram lançados 
pela CAPES programas com objetivo de ampliar o número de docentes doutores e o número de cursos de mestrado e doutorado nas regiões Norte, Centro Oeste e Nordeste, os quais passamos a discorrer nas próximas seções.

\subsection{Dinter Novas Fronteiras}

Iniciado em 2007, o Programa de Doutorado Interinstitucional - Dinter possibilitava, em nível de doutorado no país, a formação de docentes das Instituições de Ensino Superior - IES, federais ou estaduais, localizadas nas regiões Norte, Nordeste ou Centro-Oeste, via treinamento "in company", proporcionando a formação de doutores em bloco fora dos grandes centros. As IES localizadas nas Regiões Norte, Nordeste e Centro-Oeste, apresentavam solidariamente com IES que possuíam programas de pós-graduação já consolidados, propostas de Programas de Doutorado Interinstitucional, com a finalidade de capacitar recursos humanos de sua própria IES e de instituições parceiras. Como financiamento, a CAPES disponibilizava às propostas selecionadas recursos de custeio e bolsas para a formação de recursos humanos (CAPES, 2020a). A tabela 1 resume as chamadas e o número de projetos aprovados no âmbito dessa política.

Tabela 1 - Dinter Novas Fronteiras.

\section{DINTER NOVAS FRONTEIRAS 2007 - 2011}

Chamada e Editais

\begin{tabular}{c|c} 
Chamada e Editais & Projetos Aprovados \\
\hline Convite -2007 & 15 \\
\hline Edital 005/2008 & 34 \\
\hline Edital 005/2009 & 48 \\
\hline
\end{tabular}

Fonte: elaborada a partir dos Relatórios de Gestão da CAPES de 2008 e 2009; Piotto (2016).

No primeiro ano do Dinter Novas fronteiras as IES das regiões alvo foram incentivadas a participar por meio de convite enviado pela CAPES. Nos anos seguintes, foram lançados editais de seleção, sendo o último no ano de 2009 . Houve implementação de bolsas no Dinter Novas Fronteiras de 2008 a 2011, sendo 2010 o ano de maior concessão de bolsas, com 1496 benefícios (GEOCAPES, 2020). Atualmente, o programa encontra-se encerrado (Piotto, 2016).

\subsection{Programa Nacional de Cooperação Acadêmica - Novas Fronteiras (PROCAD Novas Fronteiras)}

Criado em 2007, o PROCAD Novas Fronteiras visava a consolidação de programas de pós-graduação jovens. Tinha como objetivo apoiar projetos conjuntos de ensino e pesquisa, em instituições distintas, envolvendo diferentes equipes de pesquisa, no intuito de incentivar a formação pós-graduada, a mobilidade docente e discente e a fixação de pesquisadores doutores nas Regiões Norte, Nordeste e Centro-Oeste. O financiamento da CAPES envolvia recursos de custeio, bolsa (modalidades mestrado sanduíche, doutorado sanduíche e estágio pós-doutoral), além de auxílio moradia no país (CAPES, 2010). Na Tabela 2, estão dispostas informações detalhadas dessa ação. 
Tabela 2 - PROCAD Novas Fronteiras.

\begin{tabular}{|c|c|c|c|c|}
\hline \multicolumn{5}{|c|}{ PROCAD NOVAS FRONTEIRAS 2007 - 2009} \\
\hline \multicolumn{5}{|c|}{ Chamada e Editais } \\
\hline \multicolumn{5}{|c|}{ Convite 2007} \\
\hline \multicolumn{5}{|c|}{ Edital PROCAD-NF Nº 08/ 2008} \\
\hline \multicolumn{5}{|c|}{ Edital PROCAD-NF N²1/ 2009} \\
\hline \multicolumn{5}{|c|}{ Dados Quantitativos } \\
\hline Ano & Projetos Aprovados & Equipes & Bolsas Concedidas & Investimentos $(\mathbf{R} \$)$ \\
\hline 2007 & 82 & 185 & 271 & $19.186 .811,98$ \\
\hline 2008 & 110 & 227 & 421 & $22.015 .709,49$ \\
\hline 2009 & 126 & 325 & 1.553 & $34.938 .596,90$ \\
\hline
\end{tabular}

Fonte: Elaborada a partir de Moura (2016).

No âmbito do PROCAD Novas Fronteiras houve três chamamentos públicos: o primeiro em 2007, convidando as IES localizadas nas Regiões Norte, Nordeste e Centro-Oeste, detentoras de programas de pós-graduação stricto sensu a apresentarem propostas e nos dois anos seguintes, dois editais (2008 e 2009) foram lançados. A partir das informações constantes na Tabela 2, é possível observar a ampliação do número de projetos aprovados e apoiados, além dos investimentos a cada nova chamada. Ressalta-se que esse programa se encontra encerrado.

\subsection{Programa de Formação Doutoral Docente - Prodoutoral}

O Prodoutoral foi instituído pela Portaria CAPES $n^{\circ} 112$, de 10 de agosto de 2008, e foi desenvolvido para estimular, em nível de doutorado, a qualificação dos docentes das Instituições Federais de Ensino Superior - IFES, com vistas a formar novos grupos de pesquisa em áreas estratégicas e prioritárias, a consolidar grupos já existentes, a fomentar a cooperação acadêmica, a criar programas de pós-graduação e a consolidar programas já existentes. O Programa é realizado de forma compartilhada entre a CAPES e as IFES participantes por meio de um planejamento, cujas bases são descritas no documento Plano Institucional de Formação de Quadros Docentes - Planfor, no qual a instituição solicitante estabelece os princípios, objetivos e as metas relacionados ao plano de formação de quadros docentes definidos no âmbito do Programa, considerando um período de abrangência de cinco anos. A CAPES disponibiliza bolsas de doutorado e auxílio moradia no valor de R\$1.100,00.

Assim, um docente de uma IFES participantes ao ser selecionado e liberado para cursar doutorado em um programa de pós-graduação stricto sensu em outra instituição poderia receber bolsa e auxílio moradia, se enquadrado nos objetivos estabelecidos no Planfor, incentivado a formação e a mobilidade (CAPES, 2010).

No intuito de aprimorar o Programa Prodoutoral, no ano de 2013, foi aprovado novo regulamento modificando algumas regras de funcionamento para os projetos aprovados a partir da publicação da Portaria $\mathrm{n}^{\circ} 140$, de 02 de outubro de 2013 . Na tabela 3 detalhamos as chamadas, os editais e o número de IES atendidas. 
Tabela 3 - Prodoutoral.

\begin{tabular}{c|c}
\hline \multicolumn{2}{c}{ PRODOUTORAL 2008 - Atual } \\
\hline Chamadas e Editais & IES atendidas \\
\hline 2008 & 43 \\
\hline Edital 34/2010 & 17 \\
\hline 2013 & 47 \\
\hline
\end{tabular}

Fonte: Elaborado a partir do Relatório de Gestão da CAPES de 2008; CAPES, (2010b).

Verifica-se dois chamamentos e um edital no âmbito do Prodoutoral. O programa continua vigente com bolsistas ativos. Até o ano de 2020, no Sistema de Controle de Bolsas e Auxílios - SCBA da CAPES foram registrados um total de 1.222 bolsistas que participaram do Prodoutoral, dos quais 946 foram titulados (dados cedidos pela Diretoria de Programas e Bolsas no País da CAPES).

\subsection{Dinter}

No ano de 2011, foi lançada a reedição do programa Dinter, sem a nomenclatura novas fronteiras, permitindo a participação de IES de todas as regiões do país. A CAPES manteve como financiamento das propostas selecionadas recursos de custeio e bolsas para a formação de recursos humanos.

Tabela 4 - Dinter.

\begin{tabular}{c|c}
\hline \multicolumn{2}{c}{ DINTER 2011 - Atual } \\
\hline Editais & Projetos Aprovados \\
\hline Edital 013/2011 & 34 \\
\hline Edital 013/2012 & 49 \\
\hline Edital 023/2014 & 47 \\
\hline Edital 011/2015 & 23 \\
\hline Edital 002/2016 & 23 \\
\hline
\end{tabular}

Fonte: Elaborada a partir dos Relatórios de Gestão da CAPES 2011, 2015 e 2016.

Ao todo, foram lançados cinco editais para o programa Dinter, o último no ano de 2016. Atualmente, ainda há turmas e bolsistas do Dinter ativos, portanto, o programa continua vigente, apesar do último edital ter sido lançado em 2016. Até o ano de 2020, no SCBA da CAPES foram registrados um total de 2.010 bolsistas que participaram do Dinter, com 1.370 titulados (dados cedidos pela Diretoria de Programas e Bolsas no País da CAPES).

\subsection{Programa Nacional de Cooperação Acadêmica na Amazônia (PROCAD Amazônia)}

Lançado no ano de 2018, o PROCAD Amazônia objetivava apoiar projetos conjuntos de ensino e pesquisa, em instituições distintas, que aprimorassem a formação pós-graduada com vistas à melhoria das notas de programas de pósgraduação nota 3, vinculados às IES ou Institutos de Pesquisa localizados nos Estados da Região Norte e no Estado do Maranhão. O financiamento da CAPES previa recursos de custeio, bolsa (modalidade estágio pós-doutoral no país, doutorado sanduíche no exterior, professor visitante no exterior), além de auxílio moradia no país (CAPES, 2018a). 
Tabela 5 - PROCAD Amazônia.

\begin{tabular}{c|c}
\hline \multicolumn{2}{|c}{ PROCAD Amazônia 2018 - Atual } \\
\hline \multicolumn{2}{c}{ Edital } \\
\hline Ano & Edital n $^{\circ} 21 / 2018$ \\
\hline 2018 & Projetos Aprovados \\
\hline
\end{tabular}

Fonte: Elaborado a partir de CAPES (2018a).

O programa encontra-se vigente e houve apenas um edital lançado com 79 projetos aprovados e apoiados.

\subsection{Programa Professor Visitante Nacional Sênior (PVNS - Amazônia)}

Iniciado no ano de 2018, no intuito de propiciar a professores pesquisadores de consagrado mérito científico e reconhecida experiência acadêmica a realização de estudos, participação no desenvolvimento de planos, programas e projetos que contribuíssem para o aprimoramento e a consolidação do desempenho científico-acadêmico das instituições com programas de pós-graduação pertencentes aos estados da Região Norte (Acre, Amapá, Amazonas, Pará, Rondônia, Roraima e Tocantins) e do Estado do Maranhão. O financiamento da CAPES ocorreu por meio da concessão de bolsas durante um período de dois anos, prorrogável por igual período (CAPES, 2018b).

Tabela 6 - PVNS Amazônia.

\begin{tabular}{c|c|c|c}
\hline \multicolumn{4}{c}{ PVNS - Amazônia 2018 - Atual } \\
\hline \multicolumn{4}{c}{ Edital } \\
\hline Ano & IES selecionadas & Total de Cotas de Bolsas Concedidas & Valor da Bolsa \\
\hline \multirow{2}{*}{2018} & 15 & 36 & $\begin{array}{c}\text { R } \$ 8.905,42 \text { mensais durante um } \\
\text { período de } 2 \text { anos }\end{array}$ \\
\hline
\end{tabular}

Fonte: Elaborado a partir de CAPES (2018b).

Os professores visitantes contribuem com sua experiência e conhecimento para aprimoramento dos programas da região da Amazônia. Como a bolsa é de dois anos prorrogável por mais dois o programa continua vigente. Houve apenas um edital com 15 IES selecionadas e 36 cotas de bolsas concedidas.

\subsection{Programa de Desenvolvimento da Pós-Graduação - PDPG Amazônia Legal}

Iniciativa recente, de 2020, o PDPG - Amazônia Legal objetiva fomentar Planos de Desenvolvimento de Programas de Pós-Graduação, em áreas estratégicas, localizadas na região da Amazônia Legal, no intuito de consolidar os programas de pósgraduação, nuclear novas áreas de concentração ou criar programas de pós-graduação na Amazônia Legal. Os planos devem ser propostos pelos Pró-Reitores de Pesquisa e Pós-Graduação das instituições e estarem alinhados a uma das áreas temáticas consideradas estratégicas no Edital de lançamento de seleção, de no 13/2020. As áreas são estas: biotecnologia; biodiversidade, conservação e recuperação ambiental; saúde pública, doenças tropicais e tecnologias para o trabalho em saúde; combate e prevenção voltados ao enfrentamento de epidemias; engenharias, tecnologia de informação e comunicação; clima, energia e recursos hídricos; produção animal e vegetal sustentável e diversidade sociocultural, sustentabilidade e atividades socioeconômicas. O financiamento da CAPES se dá por meio da concessão de bolsas e de recursos de custeio (CAPES, 2020a). 
Tabela 7 - PDPG - Amazônia Legal.

\begin{tabular}{|c|c|c|c|}
\hline \multicolumn{4}{|c|}{ PDPG - Amazônia Legal 2020 - Atual } \\
\hline \multicolumn{4}{|c|}{ Edital } \\
\hline \multicolumn{4}{|c|}{ Edital $n^{\circ} 13 / 2020$} \\
\hline \multicolumn{4}{|c|}{ Dados Quantitativos } \\
\hline Ano & IES selecionadas & Projetos selecionados & Financiamento \\
\hline 2020 & 27 & 67 & Até $\mathrm{R} \$ 627.200,00$ por proposta \\
\hline
\end{tabular}

Fonte: Elaborado a partir de CAPES (2020a).

O PDPG - Amazônia Legal é uma nova concepção de indução voltada a áreas estratégicas para a região da Amazônia Legal. Nessa chamada, conforme pode-se observar na Tabela 7 foram apoiados 67 projetos envolvendo 27 IES.

\subsection{Programa de Desenvolvimento da Pós-Graduação - PDPG Parcerias Estratégicas nos Estados}

Instituído pela Portaria CAPES n 131, de 3 de setembro de 2020, o PDPG - Parcerias Estratégicas nos Estados" tem como objetivo desenvolver e fortalecer a pós-graduação e a pesquisa nos Estados da Federação. Em sintonia com áreas prioritárias de âmbito regional, definidas pelas Fundações Estaduais de Amparo à Pesquisa - FAPs, os objetivos específicos apresentados no edital são: promover o desenvolvimento de programas de pós-graduação emergentes; apoiar programas de pósgraduação em consolidação; e apoiar o desenvolvimento de programas de pós-graduação consolidados, todos em áreas prioritárias de âmbito regional (CAPES, 2020c).

A portaria de instituição do PDPG - Parcerias Estratégicas nos Estados definiu os conceitos, citados nos objetivos específicos, e que também serão adotados para fins deste trabalho, como:

- $\quad$ emergentes - programas de pós-graduação stricto sensu recomendados pela CAPES criados a partir de 2013 e que passaram por apenas 1 (um) ciclo avaliativo;

- $\quad$ em consolidação - programas de pós-graduação stricto sensu, recomendados pela CAPES, que tenham permanecido com nota igual ou inferior a 4, consecutivamente, nos últimos ciclos de avaliação;

- consolidado - programas de pós-graduação stricto sensu recomendados pela CAPES, que tenham recebido nota igual ou superior a 5 no último ciclo de avaliação;

- áreas prioritárias - lacunas e potencialidades para a formação de recursos humanos e pesquisas para o desenvolvimento científico, tecnológico e de inovação no estado (CAPES, 2020c).

No PDPG - Parcerias Estratégicas no Estados, as FAPs, vinculadas ao Conselho Nacional de Fundações de Amparo à Pesquisa - CONFAP, apresentam para seleção Planos de Desenvolvimento dos Programas de Pós-Graduação stricto sensu com até 4 (quatro) projetos de apoio aos programas de pós-graduação, emergentes ou em consolidação no estado. O apoio da CAPES ocorre na forma da concessão de bolsas de estudo e auxílio financeiro (CAPES, 2020b). 
Tabela 8 - PDPG - Parcerias Estratégicas nos Estados.

\begin{tabular}{|c|c|}
\hline \multicolumn{2}{|c|}{ PDPG - Parcerias Estratégicas nos Estados 2020 - Atual } \\
\hline \multicolumn{2}{|c|}{ Edital } \\
\hline \multicolumn{2}{|c|}{ Edital n $18 / 2020$} \\
\hline \multicolumn{2}{|c|}{ Dados quantitativos } \\
\hline \multicolumn{2}{|c|}{ Ano 2020} \\
\hline Fundações selecionadas & Projetos selecionados \\
\hline 20 & 73 \\
\hline Financiamento para as Fundações & Financiamento para os Projetos \\
\hline Até R\$ 6.254.400,00 & Até R\$ $1.563 .600,00$ \\
\hline
\end{tabular}

Fonte: Elaborado a partir de CAPES (2020b).

No Edital $n^{\circ}$ 18/2020 foram aprovados os planos de 20 Fundações totalizando 73 projetos selecionados.

Nota-se que dos três programas existentes à época da divulgação do PNPG 2011/2020 (Dinter Novas Fronteiras, PROCAD Novas Fronteiras e Prodoutoral), somente uma iniciativa continua vigente na sua forma original, o Prodoutoral; os outros foram encerrados.

No caso do Dinter Novas Fronteiras, ele foi encerrado no ano de 2011, mas foi reformulado com o nome Dinter, de forma a abranger todas as regiões do país, e encontra-se ativo. Dois programas foram lançados em 2018: o PROCAD Amazônia e o PVNS Amazônia; e mais dois em 2020: o PDPG - Amazônia Legal e PDPG - Parcerias Estratégicas nos Estados, que estão em execução, e, portanto, vigentes.

É pertinente destacar que O PDPG - Amazônia Legal e o PDPG - Parcerias Estratégicas nos Estados, últimos que foram lançados, induzem as pró-reitorias a planejarem o desenvolvimento da pós-graduação em suas instituições, bem como as FAPs a planejarem o desenvolvimento da pós-graduação em seus respectivos estados, uma vez que necessitam elaborar um plano de desenvolvimento para participação nos programas.

Essa concepção é diferente dos programas anteriormente existentes que focavam na mobilidade e nas parcerias entre instituições como forma de redução de assimetrias. Esses novos programas de estímulo à pós-graduação parecem promissores, uma vez que a obrigação de apresentação de um plano leva à reflexão sobre as fragilidades e prioridades das Instituições e Estados, podendo culminar como sendo boas estratégias para a redução das assimetrias locais. Todavia, também requerem novas dinâmicas relacionais, com vistas a novas conquistas no quadro da pós-graduação nacional, o que só poderá ser mensurado em estudos futuros que busquem avaliar o impacto dos programas enquanto política pública de redução de assimetrias na pósgraduação.

\section{Panorama da Pós-Graduação na Amazônia Legal}

Observando a distribuição dos programas de pós-graduação no Brasil, verificamos que a região Sudeste detém 42,9\% dos programas, o Sul possui 21,5\%, o Nordeste 20,8\%, o Centro-Oeste $8,6 \%$ e o Norte $6,2 \%$, tendo, portanto, a menor quantidade de PPGs. Recortando os dados relativos à região da Amazônia Legal, verificamos, no ano de 2020, 421 programas de pósgraduação avaliados e recomendados pela CAPES, conforme demonstra a Tabela 9, disposta na sequência. 
Tabela 9 - Panorama de pós-graduação na região da Amazônia Legal.

\begin{tabular}{c|c|c}
\hline \multicolumn{2}{c}{ Programas de Pós-Graduação } \\
\hline UF & Região & Quantidades de PPGs \\
\hline Acre & Norte & 15 \\
\hline Amapá & Norte & 63 \\
\hline Amazonas & Norte & 67 \\
\hline Maranhão & Nordeste & 67 \\
\hline Mato Grosso & Centro-Oeste & 140 \\
\hline Pará & Norte & 17 \\
\hline Rondônia & Norte & 14 \\
\hline Roraima & Norte & 28 \\
\hline Tocantins & Norte & $\mathbf{4 2 1}$ \\
\hline
\end{tabular}

Fonte: Elaborado a partir de dados da Plataforma Sucupira da CAPES, (2020).

Nota-se que o Amapá é o Estado com o menor número de programas de pós-graduação da região da Amazônia Legal, possuindo apenas 10. O Pará é o Estado que concentra o maior número de programas de pós-graduação da região, 140 ao todo. O número total de programas de pós-graduação da região, 421, é inferior à de outros Estados do Brasil como Minas Gerais, com 479 programas, e o Rio de Janeiro, com 516 programas, por exemplo, o que evidencia, mais uma vez, o aspecto das assimetrias regionais (Plataforma Sucupira, 2020).

Outra característica a evidenciar com os dados selecionados é que a maior parte dos 421 programas de pós-graduação da Amazônia Legal são emergentes ou em consolidação, sendo apenas 35 programas de pós-graduação já consolidados (Tabela $10)$. 
Research, Society and Development, v. 10, n. 14, e26101421598, 2021

(CC BY 4.0) | ISSN 2525-3409 | DOI: http://dx.doi.org/10.33448/rsd-v10i14.21598

Tabela 10 - Programas de Pós-Graduação da Amazônia Legal por nota de avaliação CAPES.

\begin{tabular}{|c|c|c|c|c|c|c|c|}
\hline UF & Aprovado & Nota 3 & Nota 4 & Nota 5 & Nota 6 & Nota 7 & Total \\
\hline Acre & 5 & 6 & 4 & $\mathbf{0}$ & $\mathbf{0}$ & $\mathbf{0}$ & 15 \\
\hline Consolidado & & & & & & & 0 \\
\hline Em consolidação & & 2 & 3 & & & & 5 \\
\hline Emergente & 5 & 4 & 1 & & & & 10 \\
\hline Amapá & 3 & 6 & 1 & & & & 10 \\
\hline Consolidado & & & & & & & 0 \\
\hline Em consolidação & & 2 & 1 & & & & 3 \\
\hline Emergente & 3 & 4 & & & & & 7 \\
\hline Amazonas & 8 & 31 & 18 & 5 & 1 & & 63 \\
\hline Consolidado & & & & 5 & 1 & & 6 \\
\hline Em consolidação & & 19 & 15 & & & & 34 \\
\hline Emergente & 8 & 12 & 3 & & & & 23 \\
\hline Maranhão & 18 & 32 & 13 & 3 & 1 & & 67 \\
\hline Consolidado & & & & 3 & 1 & & 4 \\
\hline Em consolidação & & 17 & 10 & & & & 27 \\
\hline Emergente & 18 & 15 & 3 & & & & 36 \\
\hline Mato Grosso & 8 & 33 & 21 & 5 & & & 67 \\
\hline Consolidado & & & & 5 & & & 5 \\
\hline Em consolidação & & 18 & 20 & & & & 38 \\
\hline Emergente & 8 & 15 & 1 & & & & 24 \\
\hline Pará & 24 & 57 & 40 & 15 & 4 & & 140 \\
\hline Consolidado & & & & 15 & 4 & & 19 \\
\hline Em consolidação & & 22 & 33 & & & & 55 \\
\hline Emergente & 24 & 35 & 7 & & & & 66 \\
\hline Rondônia & 5 & 7 & 5 & & & & 17 \\
\hline Consolidado & & & & & & & 0 \\
\hline Em consolidação & & 4 & 4 & & & & 8 \\
\hline Emergente & 5 & 3 & 1 & & & & 9 \\
\hline Roraima & 3 & 8 & 3 & & & & 14 \\
\hline Consolidado & & & & & & & 0 \\
\hline Em consolidação & & 4 & 3 & & & & 7 \\
\hline Emergente & 3 & 4 & & & & & 7 \\
\hline Tocantins & 3 & 18 & 6 & 1 & & & 28 \\
\hline Consolidado & & & & 1 & & & 1 \\
\hline Em consolidação & & 8 & 5 & & & & 13 \\
\hline Emergente & 3 & 10 & 1 & & & & 14 \\
\hline Total & 77 & 198 & 111 & 29 & 6 & $\mathbf{0}$ & 421 \\
\hline
\end{tabular}

Fonte: Elaborado a partir de dados da Plataforma Sucupira CAPES, (2020).

Ao observamos as notas da avaliação dos programas do pós-graduação da região da Amazônia Legal verifica-se que a maior parte possui notas 3 e 4, extratos iniciais, demonstrando a necessidade de desenvolvimento dos programas. Apenas seis 
programas de pós-graduação são considerados de excelência, possuindo nota 6 e nenhum tem a maior nota da avaliação, ou seja, nota 7.

A partir de 2004, os programas institucionais da CAPES de bolsas e concessão de recursos de custeio para atividades acadêmicas passaram a ser agrupados em dois grupos: programas tradicionais e programas indutores e especiais. Entre os programas tradicionais, merece destaque o Programa de Demanda Social - DS, o mais antigo, maior e principal instrumento de apoio aos PPGs das instituições públicas de todo o país. Entre os programas indutores e especiais foi criado o Programa de Excelência Acadêmica - PROEX (Martins et al., 2012). O PROEX é voltado especificamente ao atendimento dos programas notas 6 e 7, objetivando manter o padrão de qualidade dos programas de pós-graduação considerados de excelência. Os PPGs quando avaliados pela CAPES com nota 6 e 7, são convidados a ingressarem no PROEX. Inseridos no PROEX recebem uma dotação orçamentária que pode ser utilizada de acordo com prioridades estabelecidas pelos próprios PPGs, em qualquer das modalidades de apoio concedidas pela CAPES, obtendo maior autonomia na gestão dos recursos.

Observando a evolução do número de programas de pós-graduação da região da Amazônia Legal no PROEX, notamos que somente a partir de 2008 houve a participação de um programa da região da Amazônia Legal, situado no Estado do Pará (Tabela 11).

Tabela 11 - Programas de Pós-Graduação da Região da Amazônia Legal no PROEX.

\begin{tabular}{c|c|c|c|c|c|c|c|c|c}
\hline \multicolumn{10}{c}{ Programas de Pós-Graduação da Região da Amazônia Legal no PROEX } \\
\hline Estados & Sigla & $\mathbf{2 0 0 4}$ & $\mathbf{2 0 0 5}$ & $\mathbf{2 0 0 8}$ & $\mathbf{2 0 1 1}$ & $\mathbf{2 0 1 4}$ & $\mathbf{2 0 1 5}$ & $\mathbf{2 0 1 8}$ & $\mathbf{2 0 1 9}$ \\
\hline Acre & AC & 0 & 0 & 0 & 0 & 0 & 0 & 0 & 0 \\
\hline Amapá & AP & 0 & 0 & 0 & 0 & 0 & 0 & 0 & 0 \\
\hline Amazonas & AM & 0 & 0 & 0 & 0 & 0 & 1 & 1 & 1 \\
\hline Mato Grosso & MT & 0 & 0 & 0 & 0 & 0 & 0 & 0 & 0 \\
\hline Pará & PA & 0 & 0 & 1 & 1 & 1 & 2 & 4 & 4 \\
\hline Rondônia & RO & 0 & 0 & 0 & 0 & 0 & 0 & 0 & 0 \\
\hline Roraima & RR & 0 & 0 & 0 & 0 & 0 & 0 & 0 & 0 \\
\hline Tocantins & TO & 0 & 0 & 0 & 0 & 0 & 0 & 0 & 0 \\
\hline Maranhão & MA & 0 & 0 & 0 & 0 & 0 & 1 & 1 & 1 \\
\hline \multicolumn{1}{c|}{ Total } & & $\mathbf{0}$ & $\mathbf{0}$ & $\mathbf{1}$ & $\mathbf{1}$ & $\mathbf{1}$ & $\mathbf{4}$ & $\mathbf{6}$ & $\mathbf{6}$ \\
\hline
\end{tabular}

Fonte: Elaborado a partir de dados do GEOCAPES (2020).

Nota-se que houve aumento do número de programas de pós-graduação da região da Amazônia Legal, no PROEX, no ano de 2015, quando ocorreu uma flexibilização da regra de ingresso, por meio de alteração regimental. Até então era necessário que o programa de pós-graduação tivesse duas avaliações CAPES consecutivas com notas 6 ou 7 para poder participar do PROEX. Em 2015 a regra foi alterada para apenas 1 avaliação com nota 6 ou 7 (CAPES, 2011). Visando a um detalhamento melhor, na Tabela 12 focalizamos os programas de pós-graduação de excelência na região da Amazônia Legal. 
Tabela 12 - Programas de Pós-graduação de Excelência na Amazônia Legal.

\begin{tabular}{c|c|c|c|c}
\hline $\begin{array}{c}\text { Ano de Ingresso no } \\
\text { PROEX }\end{array}$ & UF & IES & $\begin{array}{c}\text { Status } \\
\text { Jurídico }\end{array}$ & Nome do Programa de Pós-Graduação \\
\hline 2008 & PA & UFPA & Federal & GEOLOGIA E GEOQUÍMICA \\
\hline 2015 & MA & UFMA & Federal & POLÍTICAS PÚBLICAS \\
\hline 2015 & AM & INPA & Federal & BIOLOGIA (ECOLOGIA) \\
\hline 2015 & PA & UFPA & Federal & GENÉTICA E BIOLOGIA MOLECULAR \\
\hline 2018 & PA & UFPA & Federal & $\begin{array}{c}\text { BIOLOGIA DE AGENTES INFECCIOSOS E } \\
\text { PARASITÁRIOS }\end{array}$ \\
\hline 2018 & PA & UFPA & Federal & DESENVOLVIMENTO SUSTENTÁVEL DO TRÓPICO \\
\hline
\end{tabular}

Fonte: Elaborado a partir de dados do GEOCAPES (2020).

Constata-se que todos os programas de pós-graduação de excelência da região estão em Instituições Federais de Ensino. O Estado do Pará, concentra quatro dos seis programas de pós-graduação de excelência da região, o que resulta no registro de assimetrias, também, entre as instituições da região.

\section{Considerações Finais}

O Brasil possui dimensões continentais, tendo as regiões do país evoluído de formas distintas, por possuírem características e peculiaridades próprias que ressaltam as diferenças inter-regionais. A CAPES, em face de seus propósitos institucionais, vem ao longo dos anos desenvolvendo diversos programas voltados à redução das assimetrias na pós-graduação, em especial a regional.

É possível notar que, apesar dos esforços envidados pelos diferentes programas ao longo do tempo, as assimetrias continuam acentuadas. No ano de 2020, a CAPES lançou dois novos programas de fomento, o PDPG - Amazônia Legal e o PDPG - Parcerias Estratégicas nos Estados, com o intuito de fomentar o desenvolvimento regional e reduzir assimetrias, com uma nova lógica de indução por meio de planos de desenvolvimento.

Essas ações poderão trazer novas contribuições para a consolidação e atingimento da excelência dos programas de pósgraduação da região da Amazônia Legal, porque são iniciativas de caráter inédito e possibilitam aos atores regionais (dirigentes de IES e FAPs) maior autonomia na definição de temas e áreas prioritárias para suas regiões.

A região da Amazônia Legal, importante nacional e internacionalmente pelas suas riquezas naturais e de produção, possui um baixo índice de programas de pós-graduação em comparação com as demais regiões. Ressalta-se que dos 421 programas de pós-graduação existentes em 2020, 18,3\% são recentes, 73,4\% são emergentes ou em consolidação e apenas 8,3\% são consolidados, mostrando a necessidade de políticas públicas de fomento. Ainda que com índices menores, a pós-graduação na região vem, mesmo que timidamente, se desenvolvendo, o que pode ser constatado pela porcentagem considerável (18,3\%) de novos programas e pela participação atual de 6 programas de pós-graduação no PROEX.

$\mathrm{Na}$ apresentação dos dados quantitativos, observa-se o protagonismo do Estado do Pará e, mais precisamente, da Universidade Federal do Pará - UFPA na região, sendo o Estado com o maior número de programas de pós-graduação e a Universidade com o maior número de programas de excelência.

Trabalhos futuros poderão avaliar o impacto das recentes políticas públicas de redução de assimetrias na pós-graduação, baseadas na formulação de planos de desenvolvimento, conduzidas pela CAPES e sua efetividade na redução das desigualdades regionais. 


\section{Referências}

Brasil. (2008). Ministério da Educação. CAPES. Portaria CAPES n ${ }^{\circ} 112$, de 01 de abril de 2008. Institui e aprova o Programa de Formação Doutoral Docente Prodoutoral. http://cad.capes.gov.br/ato-administrativo-detalhar?idAtoAdmElastic=767\#anchor.

Brasil. (2013). Ministério da Educação. CAPES. Portaria CAPES nº 140, de 02 de outubro de 2013. Aprova o Regulamento do Programa de Formação Doutoral Docente - Prodoutoral. http://cad.capes.gov.br/ato-administrativo-detalhar?idAtoAdmElastic=498\#anchor.

Brasil. (2018a). Ministério da Educação. Portaria MEC n ${ }^{\circ} 321$, de 05 de abril de 2018. Dispõe sobre a avaliação da pós-graduação stricto sensu. http://cad.capes.gov.br/ato-administrativo-detalhar?idAtoAdmElastic=123.

Brasil. (2008). Ministério da Educação. História e Missão. Brasília, DF: CAPES, 2008. https://www.gov.br/capes/pt-br/acesso-ainformacao/institucional/historia-e-missao.

Brasil. (2008). Ministério da Educação. CAPES. Relatório de Gestão 2008. Brasília, DF: CAPES, 2008. https://www.gov.br/capes/pt-br/centrais-deconteudo/Relatorio_de_gestao_2008_CAPES_final31_03_com_ANEXOS.pdf.

Brasil. (2009). Ministério da Educação. CAPES. Relatório de Gestão 2009. Brasília, DF: CAPES, 2009. https://www.gov.br/capes/pt-br/centrais-deconteudo/CAPES_Relatorio_Gestao2009_Final.pdf.

Brasil. (2010). Ministério da Educação. Plano Nacional de Pós-Graduação - PNPG 2011-2020. Brasília, DF: CAPES, 2010.

Brasil. (2010b). Ministério da Educação. Programa de formação doutoral docente - Prodoutoral. Brasília, DF: CAPES, 2010b. https://www.gov.br/capes/ptbr/acesso-a-informacao/acoes-e-programas/bolsas/bolsas-no-pais/prodoutoral.

Brasil. (2011). Ministério da Educação. Relatório de Gestão 2011. Brasília, DF: CAPES, 2011. https://www.gov.br/capes/pt-br/centrais-de conteudo/relatorio_gestao_2011.pdf>.

Brasil. (2011). Ministério da Educação. CAPES. Programa de Excelência Acadêmica (PROEX). Brasília, DF: CAPES, 2011. https://www.gov.br/capes/ptbr/acesso-a-informacao/acoes-e-programas/bolsas/bolsas-no-pais/proex.

Brasil. (2014a). Ministério da Educação. CAPES. Sobre a avaliação. Brasília, DF: CAPES, 2014. https://www.gov.br/capes/pt-br/acesso-a-informacao/acoes-eprogramas/avaliacao/sobre-a-avaliacao/avaliacao-o-que-e/sobre-a-avaliacao-conceitos-processos-e-normas/conceito-avaliacao.

Brasil. (2014b). Ministério da Educação. CAPES. Comunicado CAPES - Período de Avaliação do SNPG. Brasília, DF: CAPES, 2014. https://www.gov.br/capes/pt-br/assuntos/noticias/comunicado-capes-periodo-de-avaliacao-do-snpg.

Brasil. (2015). Ministério da Educação. CAPES. Relatório de Gestão 2015. Brasília, DF: CAPES, 2015. https://www.gov.br/capes/pt-br/centrais-deconteudo/2015_Relatorio_de_Gestao.pdf.

Brasil. (2016). Ministério da Educação. CAPES. Relatório de Gestão 2016. Brasília, DF: CAPES, 2016. https://www.gov.br/capes/pt-br/centrais-deconteudo/Relatorio_de_Gestao_CAPES_2016.pdf.

Brasil. (2016). Ministério da Educação. CAPES. Dinter. Brasília, DF: CAPES, 2016. https://www.gov.br/capes/pt-br/acesso-a-informacao/acoes-eprogramas/bolsas/bolsas-no-pais/dinter.

Brasil. (2016). Ministério da Educação. CAPES. Plataforma Sucupira. Brasília, DF: CAPES, 2016. https://sucupira.capes.gov.br/sucupira/.

Brasil. (2017). Ministério da Educação. CAPES. Programas Encerrados - Dinter Novas Fronteiras. Brasília, DF: CAPES, 2017. https://www.gov.br/capes/ptbr/acesso-a-informacao/acoes-e-programas/bolsas/bolsas-no-pais/programasencerradosnopais/programasencerradosnopais.

Brasil. (2018a). Ministério da Educação. CAPES. Programa Nacional de Cooperação Acadêmica na Amazônia. Brasília, DF: CAPES, 2018. https://www.gov.br/capes/pt-br/acesso-a-informacao/acoes-e-programas/bolsas/programas-estrategicos/desenvolvimento-regional/procad-amazonia.

Brasil. (2018b). Ministério da Educação. CAPES. Programa Professor Visitante Nacional Sênior na Amazônia - Edital n 20/2018. Brasília, DF: CAPES. https://www.gov.br/capes/pt-br/centrais-de-conteudo/04062018-edital-20-pvns-amazonia-versao-final-pdf.

Brasil. (2020a). Ministério da Educação. CAPES. Programa de Desenvolvimento da Pós-Graduação (PDPG) na Amazônia Legal. Brasília, DF: CAPES. https://www.gov.br/capes/pt-br/acesso-a-informacao/acoes-e-programas/bolsas/programas-estrategicos/desenvolvimento-regional/programa-de-

desenvolvimento-da-pos-graduacao-pdpg-na-amazonia-legal.

Brasil. (2020b). Ministério da Educação. CAPES. Programa de Desenvolvimento da Pós-Graduação (PDPG) Parcerias Estratégicas nos Estados. Brasília, DF: CAPES. https://www.gov.br/capes/pt-br/acesso-a-informacao/acoes-e-programas/bolsas/programas-estrategicos/desenvolvimento-regional/programa-dedesenvolvimento-da-pos-graduacao-pdpg-parcerias-estrategicas-nos-estados.

Brasil. (2020). Ministério da Educação. CAPES. GEOCAPES - Sistema de Informações Georreferenciadas. Brasília, DF: CAPES. https://geocapes.capes.gov.br/geocapes/.

Carvalho, C. P. F. \& Silva Jr., J. R. (2017). Pesquisa, pós-graduação e conhecimento-mercadoria aplicadas no Brasil. EccoS - Rev. Cient., São Paulo. 44, 2342.

França, I. A. (2012). A gestão da pós-graduação no Brasil através de seus planos nacionais: os desafios dos gestores de cursos. Revista GUAL, 5(4), 43-67.

Kato, F. B. G. \& Ferreira, L. R. (2016). A política de expansão e financiamento da pós-graduação: as diretrizes do PNPG (2011-2020) e PNE (2014-2024) RBPAE. 32(3), 677-97. 
Research, Society and Development, v. 10, n. 14, e26101421598, 2021

(CC BY 4.0) | ISSN 2525-3409 | DOI: http://dx.doi.org/10.33448/rsd-v10i14.21598

Martins, Z. M. S. et al. (2012). Aspectos e tendências relevantes para a concessão de bolsas de pós-graduação. Momento. 21(2), 9-29.

Miranda, A. C. C., Carvalho, A. V. \& Ramos, A. S. M. (2015). Portal de periódicos da CAPES: uma política pública de acesso à informação científica e tecnológica. Biblionline, 11(1). 185-204.

Moura, E. V. (2014). Avaliação do Programa Nacional de Cooperação Acadêmica - PROCAD. [Tese de Doutorado, Universidade Federal do Rio Grande FURG]. https://lume.ufrgs.br/handle/10183/147874.

Patrus, R., Shigaki, H. B. \& Dantas, D. C. (2018). Quem não conhece seu passado está condenado a repeti-lo: distorções da avaliação da pós-graduação no Brasil à luz da história da CAPES. Cad. EBAPE.BR, 16(4).

Piotto, H. B. (2014). O doutorado interinstitucional - ação novas fronteiras (dinter/nf) como política pública para formação de pessoal de nível superior. [Dissertação de Mestrado, Universidade Federal do Rio Grande - FURG]. https://www.lume.ufrgs.br/handle/10183/150163.

Rodrigues, R. O. (2014). Pós-graduação na Amazônia: o desafio de formar (em) redes. RBPG. 11(23). 19-45.

Sá-Silva, J. R.; Almeida, C. D \& Guidani, J. F. (2009). Pesquisa documental: pistas teóricas e metodológicas. Revista Brasileira de História \& Ciências Sociais. 1(1). 1-15. 